ISSN 0103-5150

Fisioter. Mov., Curitiba, v. 26, n. 3, p. 549-557, jul./set. 2013

Licenciado sob uma Licença Creative Commons

\title{
Incapacidade funcional, sintomas depressivos e dor lombar em idosos
}

\author{
Functional disability, depressive symptoms \\ and low back pain in elderly
}

\section{Vânia Ferreira de Figueiredo ${ }^{[a]}$, Leani Souza Máximo Pereira ${ }^{[b]}$, Paulo Henrique Ferreira ${ }^{[c]}$, Aline de Morais Pereira ${ }^{[\mathrm{d}]}$, Juleimar Soares Coelho de Amorim ${ }^{[\mathrm{e}]}$}

[a] Fisioterapeuta, mestre em Ciências da Reabilitação pela Universidade Federal de Minas Gerais (UFMG), professora do Curso de Fisioterapia da Pontifícia Universidade Católica de Minas Gerais (PUC Minas), Belo Horizonte, MG - Brasil, e-mail: vaniaffigueiredo@yahoo.com.br

[b] Fisioterapeuta, doutora em Ciências Biológicas pela Universidade Federal de Minas Gerais (UFMG), com pósdoutorado no The George Institute for Global Health, Sydney University (Austrália), professora associada do Departamento de Fisioterapia da Universidade Federal de Minas Gerais (UFMG), Belo Horizonte, MG - Brasil, e-mail: leanismp.bh@terra.com.br

[c] Fisioterapeuta, professor da School of Physiotherapy, Faculty of Health Sciences, University of Sydney, Sydney, NSW Australia, e-mail: paulo.ferreira@sydney.edu.au

[d] Fisioterapeuta, especialista em Fisioterapia Ortopédica e Desportiva pela Faculdade Ciências Médicas de Minas Gerais, Belo Horizonte, MG - Brasil, e-mail: alinedemorais@yahoo.com.br

[e] Fisioterapeuta, especialista em Residência Multiprofissional em Saúde do Idoso do Hospital das Clínicas da Universidade Federal de Minas Gerais, Belo Horizonte, MG - Brasil, e-mail: juleimar@yahoo.com.br

\section{Resumo}

Introdução: A dor lombar modifica e limita aspectos tanto físicos quanto psicossociais da vida do idoso, impondo muitas vezes mudanças que causam transtornos pessoais, familiares e redução da capacidade funcional. Objetivo: Verificar a prevalência de dor lombar (DL) não específica em uma amostra de idosos da comunidade e determinar a correlação existente entre as variáveis: sexo, índice de massa corpórea (IMC), incapacidade funcional e sintomas depressivos. Materiais e métodos: Participaram deste estudo 54 idosos da comunidade (72 $\pm 5,2$ anos). As características clínicas e sociodemográficas foram avaliadas por meio de questionário semiestruturado e a DL, por meio do Questionário de McGill. Para a medida de incapacidade em indivíduos com disfunções na coluna foi usado o Questionário de Roland Morris - Brasil (RM-BR). Sintomas depressivos 
e o nível geral de atividade física foram avaliados, respectivamente, por meio da versão reduzida da Escala de Depressão Geriátrica (GDS-15) e pelo Perfil de Atividade Humana. Resultados: Evidenciou-se correlação alta e positiva entre a presença de DL, incapacidade funcional (RM-BR) ( $r s=0,774 ; p=0,00$ ) e presença de sintomas depressivos (GDS-15) ( $r s=0,271 ; p=0,048$ ). Nenhuma correlação foi evidenciada entre IMC ( $r s=0,178$; $\mathrm{p}=0$,197), sexo ( $\mathrm{rs}=-0,073 ; \mathrm{p}=0,599$ ) e DL. Conclusão: Observou-se prevalência alta de DL não específica na amostra de idosos estudada. Incapacidade funcional e depressão são condições fortemente associadas com DL em idosos da comunidade; cabe, portanto, desenvolver outras abordagens a fim de alcançar melhor efetividade das propostas terapêuticas.

Palavras-chave: Idosos. Dor lombar. Depressão.

\section{Abstract}

Introduction: Low back pain modifies and limits both physical and psychosocial aspects of elderly life and often requires changes that cause disorders personal, family, reduced functional capacity. Objective: To verify the prevalence of non-specific low back pain (LBP) in a sample of community-dwelling elderly and to determine the existing correlation between the variables: sex, body mass index, functional disability and depressive symptoms. Materials and methods: Fifty-four healthy community-dwelling elderly joined this study ( $72 \pm 5.2$ years). The clinical, social and demographic characteristics were assessed through a semi-structured questionnaire and the LBP, through the McGill Pain Questionnaire. The Rolland Morris Questionnaire (RM-BR) was used to measure the disability of the subjects with spine dysfunctions. Depressive symptoms and the general level of physical activity were assessed, respectively, by using the reduced version of the Geriatric Depression Scale (GDS77-15) and the Human Activity Profile. Results: A high positive correlation was found between the presence of $D L$, functional disability (RM-BR) ( $r S=0.774$, $p=0.00$ ) and depressive symptoms (GDS-15) ( $r s=0.271, p=0.048)$. No correlation was evidenced between BMI ( $r$ s $=0.178 ; p=0.197)$, sex ( $r s=-0.073 ; p=0.599$ ) and LBP. Conclusions: High prevalence of non-specific LBP in the sample elderly studied was observed. Functional disability and depression were shown to be strongly associated with DL conditions in elderly community need to be addressed for greater effectiveness of the proposed therapeutic.

Keywords: Elderly. Low back pain. Depression.

\section{Introdução}

O perfil de saúde da sociedade é modificado pelo crescente contingente de idosos no Brasil e no mundo, uma vez que condições agudas são substituídas por condições crônicas, múltiplas, não transmissíveis e problemas associados, próprios do envelhecimento, o que aumenta a procura por serviços de saúde (1-3).

A Dor Lombar (DL) é um dos sintomas mais frequentemente relatados pelos idosos (4 - 7), entretanto, mesmo sendo identificada como um importante problema de saúde, sua prevalência é pouco conhecida na população idosa (7 - 11).

A DL é usualmente definida como uma dor, tensão ou rigidez localizada na região compreendida entre as últimas costelas e a linha glútea. Pode ser classificada como específica, quando os sintomas são causados por condições clínicas definidas, ou como não específica, quando o mecanismo da dor não é claramente definido (12). Grande parcela da população geral, entre 50 e $80 \%$, é acometida por pelo menos um episódio de DL em algum momento da vida $(5,12)$. Estudos de prevalência internacional apontam um percentual que varia de $12 \%$ a $33 \%$ (13); no Brasil, a DL manifesta-se em $63 \%$ da população e em $57,7 \%$ da população de idosos (5).

0 desenvolvimento dessa disfunção é benigno em $90 \%$ dos pacientes e suas causas possíveis são doenças inflamatórias, degenerativas, neoplásicas, defeitos congênitos, instabilidade muscular, predisposição reumática, sinais de degeneração da coluna ou dos discos intervertebrais e outras (14). A maioria dos episódios de DL ocorre espontaneamente nas atividades cotidianas (15), mas sua etiologia é multifatorial com frequente interação dos fatores intrínsecos ao indivíduo e fatores sociodemográficos e comportamentais $(6,8,12)$.

No idoso, devido a uma maior suscetibilidade a condições que reduzem sua capacidade funcional, é 
frequente a sua associação com prejuízos nas funções do corpo, dificuldades no desempenho das atividades cotidianas e restrição na participação social do indivíduo (15).

Vários estudos têm mostrado que 30-60\% dos indivíduos com DL apresentam sintomas depressivos (16 - 21). Além disso, sentimentos de depressão podem ser considerados preditores do tempo para recuperação da dor, incapacidade e trabalho $(22,23)$.

Nesse contexto, os objetivos deste estudo foram verificar a prevalência de DL não específica em uma amostra de idosos da comunidade e determinar a correlação existente entre as variáveis sexo, índice de massa corpórea (IMC), incapacidade funcional e sintomas depressivos.

\section{Materiais e métodos}

Realizou-se estudo observacional de corte transversal, com aprovação do Comitê de Ética em Pesquisa da Universidade Federal de Minas Gerais, parecer ETIC 324/07, conforme Resolução n. 196/96 do Conselho Nacional de Saúde, que trata do Código de Ética da Pesquisa envolvendo seres humanos. Cada participante, após leitura e esclarecimento dos termos do estudo, assinou o Termo de Consentimento Livre e Esclarecido, concordando em participar da pesquisa.

A amostra foi constituída por 54 idosos da comunidade, de ambos os sexos, com idade igual ou superior a 65 anos, sem alterações cognitivas identificadas por meio do Mini Exame do Estado Mental (MEEM) (24). 0 cálculo amostral, realizado previamente, seguiu os logaritmos propostos por Maxwell (25). Foram adotados como critérios de exclusão: presença de dores agudas de coluna; sinais evidentes de radiculopatias (alterações de reflexos, dermátomos e/ou miótomos, teste de elevação da perna estendida positivos); histórico positivo para doenças neurológicas, cirurgia toracoabdominal e da coluna, fraturas vertebrais e câncer; presença de bandeiras vermelhas (25); deformidades graves observáveis na coluna (escoliose, hipercifose); realização de tratamento fisioterapêutico para problemas de coluna nos últimos seis meses; prática de exercícios para musculatura estabilizadora da coluna lombar e/ou do assoalho pélvico.

Para caracterização da amostra foi utilizado um questionário contendo dados clínicos sociodemográficos e funcionais apresentados na Tabela 1.
A presença ou não de DL foi determinada a partir da resposta para a seguinte pergunta: "nos últimos doze meses, o(a) Sr.(a) sentiu dores em suas costas?" $(18,26,27,28)$. A localização da DL foi apontada pelo voluntário em uma figura do corpo humano em posição ereta, supina e dorsal $(7,29)$. Caso a resposta fosse afirmativa, o participante respondia às demais questões do Questionário de dor de McGill (BR-MPQ) (30-32). O BR-MPQ é um instrumento adequado para avaliar a dor crônica em idosos (33); índices de confiabilidade intra e interexaminador do instrumento para a versão brasileira foram de 0,86 e 0,89 para doenças ortopédicas e 0,71 e 0,68 para doenças neurológicas, respectivamente (33). Para a medida de incapacidade funcional foi usado o Questionário de Roland Morris - Brasil (RM-BR) (34-37), traduzido e adaptado culturalmente para a população brasileira (34), apresentou coeficientes de confiabilidade teste-reteste de 0,86 e interexaminador de $0,95(34,37)$.

A presença de sintomas depressivos e o nível geral de atividade física foram avaliados, respectivamente, por meio da Escala de Depressão Geriátrica, versão reduzida com 15 itens (GDS-15) (38) e pelo Perfil de Atividade Humana (PAH) (39). Almeida e Almeida (38) relataram índices de confiabilidade, sensibilidade e especificidade para a versão brasileira da GDS-15 de 0,81\%, 85,4\% e 73,9\% respectivamente, considerando o ponto de corte de cinco. Já a versão brasileira do PAH obteve um coeficiente global de consistência interna de 0,91 para sua aplicação em idosos (39).

A análise descritiva das variáveis quantitativas foi realizada empregando-se o cálculo da média, a medida de tendência central, o desvio padrão e a medida de variabilidade (dispersão) dos dados amostrais. Já as variáveis qualitativas foram obtidas a partir do cálculo de frequências das categorias utilizadas para cada uma.

Para verificar a relação entre a variável dor lombar e as variáveis relacionadas a incapacidade (RM-BR), sintomas depressivos (GDS), IMC e sexo foi realizado índice de correlação de Spearman. Foram considerados para essas análises somente aqueles idosos que afirmaram sentir dor lombar ( $\mathrm{n}=34 ; 61,8 \%$ ). Para confirmar a normalidade dos dados, foi utilizado o teste estatístico de Anderson-Darling.

Toda a análise estatística foi realizada por meio do pacote estatístico Statistical Package for the Social Science (SPSS) para Windows (versão 13.0, SPSS Inc. ${ }^{\odot}$, Chicago, Illinois), com exceção do teste de normalidade, para o qual foi utilizado o MiniTAB 
14 Statistical Software. Em todos os testes usados foi considerado como significativo $\alpha<0,05$.

\section{Resultados}

Participaram deste estudo 54 idosos, com idade média de $72( \pm 5,2)$ anos e variação de 65 a 84 anos; o sexo feminino representou $76 \%$ da amostra. A maioria era casada ou vivia com companheiro(a) $(48,1 \%)$; também se observou grande parcela de viúvos(as) (31,5\%). Cerca de 35\% dos idosos relataram renda mensal familiar de 3 a 5 salários mínimos. Sobre o nível de escolaridade dos idosos, aproximadamente metade $(48,1 \%)$ informou ter de 1 a 4 anos de estudo. Os dados sociodemográficos são apresentados na Tabela 1.

Após o cálculo do IMC dos participantes, $46,3 \%$ da amostra apresentou o padrão eutrófico $\left(22 \mathrm{a} 27 \mathrm{~kg} / \mathrm{m}^{2}\right)$, de acordo com os pontos de corte para idosos, propostos por Lipschitz (40). Com relação ao nível de atividade física, avaliado pelo PAH, os idosos foram classificados como ativos e moderadamente ativos $(66,7 \%$ e $33,3 \%$, respectivamente). Os idosos foram questionados quanto a problemas de saúde diagnosticados pelo médico e relataram comorbidades, com média de $4,78( \pm 2,35)$, sendo a hipertensão $(64,8 \%)$ a mais frequente.

Para aqueles idosos com positividade para dor lom$\operatorname{bar}(\mathrm{n}=34)$, notou-se que mais da metade $(52,9 \%)$ referiu dor lombar de intensidade moderada $(2,24 \pm 0,78)$ e 44,1\% classificaram-na como breve/momentânea/ transitória, de acordo com o questionário de BR-MPQ. Quanto à funcionalidade avaliada pelo questionário de RM-BR, o desempenho dos idosos avaliados apresentou média de 6,8 $\pm 6,4$ e mediana de 3,5 pontos. A Tabela 2 fornece uma síntese das informações dos dados relacionados aos indivíduos com dor lombar.

A interpretação dos resultados do coeficiente de correlação de Spearman pode ser evidenciada na Tabela 3. Uma correlação alta e positiva com a presença de dor lombar foi indicada tanto para a incapacidade funcional $(\mathrm{RM}-\mathrm{Br})\left(\mathrm{r}_{\mathrm{s}}=0,774 ; \mathrm{p}=0,00\right)$ quanto para presença de sintomas depressivos (GDS - 15) $\left(\mathrm{r}_{\mathrm{s}}=0,271 ; \mathrm{p}=\right.$ $0,048)$. Nenhuma correlação foi evidenciada entre IMC $\left(r_{s}=0,178 ; p=0,197\right)$, sexo $\left(r_{s}=-0,073 ; p=0,599\right)$ e DL.

\section{Discussão}

O presente estudo investigou em uma amostra de idosos com DL não específica, moradores da comunidade, a possível correlação entre características físicas (IMC), psicossociais (sintomas depressivos), sexo e função (incapacidade/RM-BR). Alguns estudos internacionais prévios, envolvendo a população idosa, reportam associação entre essas variáveis $(35,36,41,42)$, mas entre a população brasileira esses dados ainda não estão bem estabelecidos.

A amostra avaliada apresentou características clínicas e demográficas semelhantes a outros estudos populacionais que descrevem o perfil do idoso brasileiro $(43,44)$, exceto com relação à renda familiar média, que foi mais elevada, e pelo nível de atividade física (ativo e moderadamente ativo). A amostra apresentou uma prevalência maior de mulheres, casadas ou viúvas; com baixa escolaridade e alta morbidade, indicada pelo número de condições clínicas de saúde associadas, sendo que a condição de saúde mais relatada foi a hipertensão. Esses achados confirmam que as mulheres vivem mais que os homens, ficando mais tempo expostas aos fatores de risco, convivem com mais comorbidades e vivenciam a cronicidade das condições clínicas - fenômeno chamado de "feminização" da velhice (44).

Sintomas depressivos e incapacidade física têm sido temas comumente associados com dor lombar em idosos da comunidade em diversos debates (10, $18,19,21,41)$.

Correlação forte e positiva entre dor lombar e incapacidade física foi evidenciada corroborando com os achados de outros estudos, que relatam limitações em atividades diárias como realizar o autocuidado e tarefas domésticas, ir às compras, caminhar, levantar objetos etc. Também as atividades ligadas à participação social como visitar amigos e frequentar igreja se tormam mais difíceis.

A relação entre DL e sintomas depressivos parece ser uma condição bidirecional (41-48). Alguns estudos relatam que sintomas depressivos são fatores de risco para o desenvolvimento futuro de DL em pessoas que previamente não apresentavam DL $(15,21)$ enquanto outros $(17,48)$ acreditam que a depressão seja consequência. No entanto, embora não haja um consenso entre a ordem dos fatos, a relação entre essas duas condições clinicas é confirmada (16).

Na presença de depressão, devem ser considerados a cronicidade e os sintomas predominantes. Os pacientes com depressão crônica e sem relação específica com a dor e as limitações da patologia de base apresentam piores resultados em tratamento, se comparados àqueles cuja depressão está diretamente relacionada à dor. Alguns sintomas depressivos, como distúrbio do sono, 
Tabela 1 - Características sociodemográficas e clínicas da amostra $(n=54)$

\begin{tabular}{|c|c|c|c|}
\hline Características & n (\%) & Média ( \pm d.p.) & Intervalo \\
\hline Idade (anos) & & $72( \pm 5,2)$ & $65-84$ \\
\hline \multicolumn{4}{|l|}{ Sexo } \\
\hline Masculino & $13(24 \%)$ & & \\
\hline Feminino & $41(76 \%)$ & & \\
\hline \multicolumn{4}{|l|}{ Estado civil } \\
\hline $\begin{array}{l}\text { Casado(a) ou vive com } \\
\text { companheiro(a) }\end{array}$ & $26(48,1 \%)$ & & \\
\hline Solteiro(a) & $6(11,1 \%)$ & & \\
\hline $\begin{array}{l}\text { Divorciado(a), separado(a) ou } \\
\text { desquitado(a) }\end{array}$ & $5(9,3 \%)$ & & \\
\hline Viúvo(a) & $17(31,5 \%)$ & & \\
\hline $\begin{array}{l}\text { Renda familiar (salários } \\
\text { mínimos) }\end{array}$ & & $10,5( \pm 5,9)$ & $1 / 2-20$ \\
\hline Escolaridade (anos) & & $5,5( \pm 3,6)$ & Analfabeto-11 \\
\hline \multicolumn{4}{|l|}{ IMC $\left(\mathrm{kg} / \mathrm{m}^{2}\right)$} \\
\hline$<22 \mathrm{~kg} / \mathrm{m}^{2}$ & $8(14,8 \%)$ & & \\
\hline 22 a 27 kg/m² & $25(46,3 \%)$ & & \\
\hline$>27 \mathrm{~kg} / \mathrm{m}^{2}$ & $21(38,9 \%)$ & & \\
\hline $\mathrm{n}$ de comorbidades & & $4,78( \pm 2,35)$ & $1-13$ \\
\hline \multicolumn{4}{|l|}{ GDS } \\
\hline Rastreio negativo & $43(79,6 \%)$ & & \\
\hline Rastreio positivo & $11(20,4 \%)$ & & \\
\hline \multicolumn{4}{|l|}{ PAH } \\
\hline Ativo & $36(66,7 \%)$ & & \\
\hline Moderadamente ativo & $18(33,3 \%)$ & & \\
\hline Dor lombar (sim) & $34(61,8 \%)$ & & \\
\hline
\end{tabular}

Legenda: d.p. = desvio padrão; \% = porcentagem relativa ao $\mathrm{n}$ total de 54 idosos; IMC = índice de massa corporal com pontos de corte para idosos sugeridos por Lipschitz (40); GDS = escala de depressão geriátrica; PAH = perfil de atividade humana.

Fonte: Dados da pesquisa.

problemas com concentração e modificações no peso, podem ser consequência direta da dor ou das limitações físicas. A superestimação do quadro clínico pode sofrer influências de distúrbios cognitivo-comportamentais, como ansiedade e depressão $(16,20,49,50)$.

Edmond e Felson (42) evidenciaram em seu estudo que idosos da comunidade com DL relataram dificuldade de permanecer em ortostatismo por um tempo prolongado, puxar ou empurrar um objeto e caminhar uma distância aproximada de $800 \mathrm{~m}$. Leveille (45) relata que mulheres idosas com DL apresentavam maior dificuldade em realizar as atividades básicas e instrumentais de vida diária quando comparadas com mulheres assintomaticas.

Apesar de a intensidade média de dor relatada ser moderada, o nível de incapacidade física gerada foi relativamente baixo. Alguns estudos reforçam esses resultados, relatando que há uma fraca correlação 
Tabela 2 - Dados relacionados aos participantes com DL $(n=34)$

\begin{tabular}{lccc}
\hline Variáveis & $\mathbf{n}(\%)$ & Média $\mathbf{( \pm \text { d.p. } )}$ & Intervalo \\
\hline Intensidade da dor (BR-MPQ) & & $2,24( \pm 0,78)$ & $1-4$ \\
Fraca (1) & $5(14,7 \%)$ & \\
Moderada (2) & $18(52,9 \%)$ & \\
Forte (3) & $9(26,5 \%)$ & \\
Insuportável (4) & $2(5,9 \%)$ & \\
\hline Padrão temporal da dor (BR-MPQ) & & \\
Contínua/estável/constante & $10(29,4 \%)$ & \\
Ritmada/periódica/intermitente & $9(26,4 \%)$ & \\
Breve/momentânea/transitória & $15(44,1 \%)$ & $6,8( \pm 6,4)$ \\
\hline RM-BR & & \\
\hline
\end{tabular}

Legenda: d.p. = desvio padrão; \% = porcentagem relativa; BR-MPQ = questionário de dor de McGill - Brasil; RM-BR = questionário de Roland Morris - Brasil.

Fonte: Dados da pesquisa.

Tabela 3 - Correlação entre DL e as variáveis incapacidade, sintomas depressivos, IMC e sexo

\begin{tabular}{lcccccccc}
\hline Variável & \multicolumn{3}{c}{ Incapacidade (RM-Br) } & \multicolumn{2}{l}{ Sintomas depressivos } & \multicolumn{2}{c}{ Escore IMC } & \multicolumn{2}{c}{ Sexo } \\
\hline \multirow{3}{*}{ (GDS) } & $\mathrm{r}_{\mathrm{s}}$ & $\mathrm{p}$ & $\mathrm{r}_{\mathrm{s}}$ & $\mathrm{p}$ & $\mathrm{r}_{\mathrm{s}}$ & $\mathrm{p}$ & $\mathrm{r}_{\mathrm{s}}$ & $\mathrm{P}$ \\
Dor Lombar & 0,774 & $0,001^{*}$ & 0,271 & $0,048^{*}$ & 0,178 & 0,197 & $-0,073$ & 0,599 \\
\hline
\end{tabular}

Legenda: $\mathrm{RM}-\mathrm{Br}=$ questionário de Roland Morris; GDS = escala de depressão geriátrica; IMC = índice de massa corpórea; rs = valor da correlação de Spearman; * $=p<0,05$.

Fonte: Dados da pesquisa.

entre incapacidade e intensidade da dor lombar (46). Isso ocorre porque a dor é uma condição subjetiva e pessoal, sendo influenciada por fatores sociais, mentais, culturais e espirituais $(5,46)$; no caso do idoso, há maior resiliência em relação à ocorrência da dor (47).

O PAH é um questionário de autorrelato que avalia o nível funcional e de atividade física, tanto para indivíduos saudáveis, em qualquer faixa etária, quanto para aqueles com algum grau de disfunção do nível de atividade física (39). Cabe salientar que a amostra foi de conveniência e constituída de idosos da comunidade e um dos critérios de inclusão foi a capacidade de locomoção independente; nesse contexto, possivelmente esse perfil dos participantes influenciou no bom desempenho dos idosos no PAH, considerando que o questionário inclui muitas atividades de baixo nível de dificuldade para sua execução, como "ouvir rádio", "levantar de uma cadeira sem ajuda", "ler livros ou revistas", "ficar de pé por um minuto", entre outras. Por outro lado, culturalmente e diferentemente dos homens, as mulheres desenvolvem, no seu dia a dia, mais atividades consideradas como domésticas, que na maioria das vezes são de moderada a alta dificuldade.

Alguns estudos indicam o IMC elevado como fator de risco para DL $(7,14)$ e sugerem uma prevalência dessa disfunção no sexo feminino $(4,7,47)$. Entretanto, nenhuma correlação foi evidenciada no presente estudo entre IMC, sexo e DL não especifica. Quanto a essas evidências, Shiri et al. (51), em uma recente revisão sistemática para investigar a relação entre o peso corporal e DL, afirmam que o sobrepeso e a obesidade são fatores de risco potencialmente 
modificáveis para prevenir dor lombar; e Silva et al. (7) e Wadell $(52,53)$ atribuem a prevalência do sexo feminino com dor lombar tanto um achado de viés de informação, pois as mulheres apresentam melhor percepção da dor e maior procura por serviços de saúde, quanto a características biomecânicas, como menor massa óssea e muscular, menor adaptação articular durante elevação de peso e a gravidez.

Um dado importante a considerar é em relação ao uso de medicamentos para dor. Todos os participantes com sintomas dolorosos relataram utilizar medicamento analgésico apenas quando sentiam dor forte, nenhum estava em uso contínuo da medicação. Apenas três pacientes (8,8\%) relataram uso de antidepressivos, um número baixo para considerar interferência nos resultados encontrados.

0 baixo índice de incapacidade física, causada pela DL observado na amostra, provavelmente ocorreu porque foram excluídos idosos com DL em decorrência de condições clínicas prévias, como fraturas osteoporóticas, degeneração discal, compressão radicular e alterações posturais graves. Foram incluídos apenas idosos com DL não específica.

0 estudo realizado foi transversal com uma amostra de idosos da comunidade, o que limita sua validade externa.

\section{Conclusão}

Os resultados deste estudo corroboram com outras evidências de que os escores mais altos de incapacidade funcional e sintomas depressivos podem ser encontrados em pacientes idosos com lombalgia. Uma vez que o nível de incapacidade funcional não se correlaciona com a intensidade da dor, sintomas depressivos podem ser tanto fatores de risco quanto consequência da dor lombar, e a prevalência da DL no sexo feminino pode ser explicada pelas suas características biomecânicas ou vista como uma informação. No entanto, é importante apontar que os profissionais envolvidos na assistência aos idosos devem estar atentos para avaliar, interpretar as correlações entre as condições clínicas e desenvolver estratégias multifocais para prevenir impactos negativos sobre a função física, mental, comportamental e social.

Em relação aos constructos dos instrumentos e medidas utilizados, o resultado do autorrelato no Perfil de Atividade Humana (PAH) foi influenciado pela capacidade de locomoção independente. Este estudo parece ser o primeiro que estabeleceu correlação clínica entre a dor lombar, os sintomas depressivos e a incapacidade funcional em idosos brasileiros, mostrando que o diagnóstico de sintomas depressivos em pacientes com dor lombar e uma análise dos fatores relacionados são essenciais para elaborar programas específicos de intervenção e eliminar esses fatores de risco, bem como promover ações de saúde.

\section{Referências}

1. Fiedler MM, Peres KG. Capacidade funcional e fatores associados em idosos do Sul do Brasil: um estudo de base populacional. Cad Saúde Pública. 2008;24(2):409-15.

2. Alves L, Leimann B, Vasconcelos M, Carvalho M, Vasconcelos A, Fonseca T, et al. A influência das doenças crônicas na capacidade funcional dos idosos do Município de São Paulo, Brasil. Cad Saúde Pública. 2007;23(8):1924-30.

3. Lima-Costa M, Barreto S, Giatti L. Condições de saúde, capacidade funcional, uso de serviços de saúde e gastos com medicamentos da população idosa brasileira: um estudo descritivo baseado na Pesquisa Nacional por Amostra de Domicílios. Cad Saúde Pública. 2003;19(3):735-43.

4. Dellaroza M, Furuya R, Cabrera M, Matsua T, Trelha C, Yamada K, et al. Caracterização da dor crônica e métodos analgésicos utilizados por idosos da comunidade. Rev Assoc Med Bras. 2008;54(1):36-41.

5. Ferreira GD, Silva MC, Rombaldi AJ, Wrege ED, Siqueira FV, Hallal PC. Prevalência de dor nas costas e fatores associados em adultos do Sul do Brasil: estudo de base populacional. Rev bras fisioter. 2011;15(1):31-6.

6. Teixeira MJ, Teixeira WGJ, Santos F, Andrade DCA, Bezerra SL, Figueiro JB, et al. Epidemiologia clínica da dor musculo-esquelética. Rev med (São Paulo). 2001;80:1-21.

7. Silva MCD, Fassa AG, Valle NC. Dor lombar crônica em uma população adulta do Sul do Brasil: prevalência e fatores associados. Cad Saúde Pública. 2004;20(2):377-85.

8. Bressler H, Keyes W, Rochon P, Badley E. The prevalence of low back pain in the elderly. A systematic review of the literature. Spine. 1999;24(17):1813-9. 
9. Weiner DK, Sakamoto S, Pereira S, Breuer P. Chronic low back pain in older adults: prevalence, reliability, and validity of physical examination findings. J Am Geriatr Soc. 2006;54(1):11-20.

10. Hartvigsen J, Christensen K, Frederiksen H. Back pain remains a common symptom in old age. A population-based study of 4486 Danish twins aged 70-102. Eur Spine J. 2003;12(5):528-34.

11. Gallagher R. Low back pain, health status, and quality of life in older adults: challenge and opportunity. Pain Med. 2003;4(4):305-7.

12. Edmond SL, Felson DT. Prevalence of back symptoms in elders. J Rheumatol. 2000;27(1):220-5.

13. Scheele J, Luijsterburg PAJ, Ferreira ML, Maher CG, Pereira L, Peul WC, et al. Back complaints in the elders (BACE); design of cohort studies in primary care: an international consortium. BMC Musculoskelet Disord. 2011;12:193-202.

14. van Tulder M, Koes B, Bombardier C. Low back pain. Best Pract Res Clin Rheumatol. 2002;16(5):761-75.

15. Andersson GB. Epidemiological features of chronic low-back pain. Lancet. 1999;354(9178):581-5.

16. Kaptan H, Yalçin ES, Kasimcan O. Correlation of low back pain caused by lumbar spinal stenosis and depression in women: a clinical study. Arch Orthop Trauma Surg. 2012;132(7):963-7.

17. Meyer T, Cooper J, Raspe H. Disabling low back pain and depressive symptoms in the community-dwelling elderly. Spine. 2007;32(21):2380-6.

18. Weiner DK, Haggerty CL, Kritchevsky SB, Harris T, Simonsick EM, Nevitt M, et al. How does low back pain impact physical function in independent, well-functioning older adults? Evidence from the Health $\mathrm{ABC}$ cohort and implications for the future. Pain Med. 2003;4(4):311-20.

19. Weiner DK, Rudy TE, Morrow L, Slaboda J, Lieber S. The relationship between pain, neuropsychological performance, and physical function in community-dwelling older adults with chronic low back pain. Pain Med. 2006;7(1):60-70.

20. Ha JY, Kim ES, Kim HJ, Park SJ. Factors associated with depressive symptoms in patients with chronic low back pain. Ann Rehabil Med. 2011;35(5):710-8.
21. Hartvigsen J, Frederiksen H, Christensen K. Physical and mental function and incident low back pain in senior. A population-based two prospective study of 1387 twins age 70 to 100 years. Spine. 2006; 31(14):1628-32.

22. Ledoux E, Dubois J, Descarreaux M. Physical and psychosocial predictors of Functional trunk capacity in older adults with and without low back pain. J Manipulative Physiol Ther. 2012;35(5):338-45.

23. Ramond A, Bouton C, Richard I, Roquelaure Y, Baufreton C, Legrand E, et al. Psychosocial risk factors for chronic low back pain in primary care - a systematic review. Fam Pract. 2011;28(1):12-21.

24. Brucki SMD, Nitrini R, Caramelli P, Bertolucci PHF, Okamoto IH. Sugestões para o uso do mini-exame do estado mental no Brasil. Arq Neuropsiquiatr. 2003;61(3B):777-81.

25. Maxwell SE. Sample size and multiple regression analysis. Psychol Methods. 2000;5(4):434-58.

26. Cecchi F, Debolini P, Lova RM, Macchi C, Bandinelli S, Bartali B, et al. Epidemiology of back pain in a representative cohort of Italian persons 65 years of age and older: the InCHIANTI study. Spine. 2006;31(10):1149-55.

27. de Vet HC, Heymans MW, Dunn KM, Pope DP, van der Beek AJ, Macfarlane GJ, et al. Episodes of low back pain: a proposal for uniform definitions to be used in research. Spine. 2002;27(21):2409-16.

28. Hicks GE, Simonsick EM, Harris TB, Newman AB, Weiner DK, Nevitt MA, et al. Trunk muscle composition as a predictor of reduced functional capacity in the health, aging and body composition study: the moderating role of back pain. J Gerontol A Biol Sci Med Sci. 2005;60(11):1420-4.

29. Richardson C, Snijders C, Hides J, Damen L, Pas M, Storm J. The relation between the transversus abdominis muscles, sacroiliac joint mechanics, and low back pain. Spine. 2002;27(4):399-405.

30. Castro CES. A formação lingüística da dor - versão brasileira do questionário McGill de dor [tese]. São Carlos: Universidade Federal de São Carlos; 1999.

31. Pimenta CAM, Teixeira MJ. Questionário de dor McGill: proposta de adaptação para língua portuguesa. Rev Esc Enf USP. 1996;30(3):473-83. 
32. Varoli FK, Pedrazzi V. Adapted version of the McGill pain questionnaire to Brazilian Portuguese. Braz. Dent. J. 2006;17(4):328-35.

33. Santos C, Pereira L, Resende M, Magno F. Aplicação da versão brasileira do questionário de dor Mcgill em idosos com dor crônica. Acta Fisiátrica. 2006;13(2):75-82.

34. Nusbaum L, Natour J, Ferraz M, Goldenberg J. Translation, adaptation and validation of the Roland-Morris questionnaire - Brazil Roland-Morris. Braz J Med Biol Res. 2001;34(2):203-10.

35. Weiner DK, Rudy TE, Kim YS, Golla S. Do medical factors predict disability in older adults with persistent low back pain? Pain. 2004;112(1-2):214-20.

36. Weiner D, Pieper C, McConnell E, Martinez S, Keefe F. Pain measurement in elders with chronic low back pain: traditional and alternative approaches. Pain. 1996;67(2-3):461-7.

37. Costa LO, Maher CG, Latimer J, Ferreira PH, Pozzi GC, Ribeiro RN. Psychometric characteristics of the Brazilian-Portuguese versions of the Functional Rating Index and the Roland Morris Disability Questionnaire. Spine. 2007;32(17):1902-7.

38. Almeida OP, Almeida SA. Confiabilidade da versão brasileira da Escala de Depressão em Geriatria (GDS) versão reduzida. Arq Neuropsiquiatr. 1999;57(2B):421-6.

39. Souza AC, Magalhaes LC, Teixeira-Salmela LF. Adaptação transcultural e análise das propriedades psicométricas da versão brasileira do perfil de atividade humana. Cad Saúde Pública. 2006;22(12):2623-36.

40. Lipschitz D. Screening for nutritional status in the elderly. Prim Care. 1994;21(1):55-67.

41. Instituto Brasileiro de Geografia e Estatística [IBGE]. Perfil dos idosos responsáveis pelo domicílio 2000. Rio de Janeiro: IBGE; 2002.

42. Edmond SL, Felson DT. Function and back symptoms in older adults. J Am Geriatr Soc. 2003;51(12):1702-9.

43. Ramos LR. Fatores determinantes do envelhecimento saudável em idosos residentes em centro urbano: Projeto Epidoso, São Paulo. Cad Saúde Pública. 2003;19(3):793-8.

44. Lima-Costa MFF. A saúde dos adultos na região metropolitana de Belo Horizonte: um estudo epidemiológico de base populacional. Belo Horizonte: Núcleo de Estudos em Saúde Pública e Envelhecimento; 2004.
45. Leveille SG, Guralnik JM, Hochberg M, Hirsch R, Ferrucci L, Langlois J, et al. Low back pain and disability in older women: independent association with difficulty but not inability to perform daily activities. J Gerontol A Biol Sci Med Sci. 1999;54(10):M487-93.

46. Takahashi N, Kikuchi S, Konno S, Morita S, Suzukamo Y, Green J, et al. Discrepancy between disability and the severity of low back pain: demographic, psychologic, and employment-related factors. Spine. 2006;31(8):931-9.

47. Rudy TE, Weiner DK, Lieber SJ, Slaboda J, Boston JR. The impact of chronic low back pain on older adults: a comparative study of patients and controls. Pain. 2007;131(3):293-301.

48. Reid MC, Williams CS, Concato J, Tinetti ME, Gill TM. Depressive symptoms as a risk factor for disabling back pain in community-dwelling older persons. J Am Geriatr Soc. 2003;51(12):1717-2003.

49. Hill JC, Fritz JM. Psychosocial influences on low back pain, disability, and response to treatment. Phys Ther. 2011;91(5):712-21.

50. Gale CR, Aihie AS, Cooper C, Dennison EM, Starr JM, Whalley LJ, et al. Factors associated with symptoms of anxiety and depression in five cohorts of community-based older people: the HALCyon (Healthy Ageing across the Life Course) Programme. Psychol Med. 2011;41(10):2057-73.

51. Shiri R, Karppinen J, Leino-Arjas P, Solovieva S, Viikari-Juntura E. The association between obesity and low back pain: a meta-analysis. Am J Epidemiol. 2010;171(2):135-54.

52. Waddell G, editor. The back pain revolution. 2nd ed. Edinburgh: Churchill Livingstone; 2004.

53. Waddell G. Diagnostic triage. In: Waddell G, editor. The back pain revolution. 2nd ed. Edinburgh: Churchill Livingstone; 2004.

Recebido: 27/05/2012 Received: 05/27/2012

Aprovado: 06/02/2013

Approved: 02/06/2013 